\title{
Inserción laboral de personas con Diversidad Funcional Cognitiva ${ }^{1}$
}

\author{
Labor insertion of people with cognitive functional diversity
}

\author{
Maribel Camacho Vásquez * \\ María José Solís Alfaro **
}

\begin{abstract}
RESUMEN
El artículo presente se desarrollará una investigación de índole cuantitativa en acerca de la inserción dentro del campo laboral de personas con diversidad funcional cognitiva. El objetivo de la misma fue reconocer la situación laboral de personas con diversidad funcional cognitiva en Naranjo de Alajuela, desde la perspectiva de dos expertas en el cuido y trabajo con personas con diversidad funcional cognitiva, y de tres personas con diversidad funcional cognitiva. Dentro de los resultados se encontró que estas personas no perciben el apoyo ni las oportunidades necesarias para laborar, tanto de parte de reclutadores y jefaturas, como por parte de familiares. Por otra parte las expertas mencionan que las leyes que protegen a estas personas no se cumplen como se deben ya que no se perciben igual para todas las personas, sino que cada jefe la aplica según sea su conveniencia. Las leyes para la inclusión de personas con discapacidad o diversidad se adecúan mejor para personas con diversidades físicas y no tanto para diversidades cognitivas.

Palabras clave: Diversidad, diversidad funcional cognitiva, Inserción laboral, leyes.
\end{abstract}

\begin{abstract}
The present article will develop a quantitative research on the insertion within the labor field of people with cognitive functional diversity. The objective was to recognize the employment situation of people with cognitive functional diversity in Naranjo de Alajuela, from the perspective of two experts in care and work with people with cognitive functional diversity, and three people with cognitive functional diversity. Within the results it was found that these people do not perceive the support or the necessary opportunities to work, both on the part of recruiters and heads, as well as on behalf of relatives. On the other hand experts mention that the laws that protect these people are not fulfilled as they should be since they are not perceived the same for all people, because each boss applies it according to their convenience. Laws for the inclusion of people with disabilities or diversity are better suited for people with physical diversity and not so much for cognitive diversities.
\end{abstract}

Key Words: Diversity, cognitive functional diversity, labor insertion, laws.

\footnotetext{
${ }^{1}$ Investigación elaborada en el Módulo de Psicología de los Procesos Laborales, Universidad de Costa Rica, Sede de Occidente, bajo la supervisión de la Licda. Harlen Alpízar Rojas. Correo electrónico: harlen.alpizarroja@ucr.ac.cr

* Universidad de Costa Rica, Sede de Occidente. Estudiante de Psicología. Costa Rica.

Correo electrónico: maribellcamacho@gmail.com

** Universidad de Costa Rica, Sede de Occidente. Estudiante de Psicología. Costa Rica. Correo electrónico: mariajose090494@gmail.com.
}

Recepción: 23/5/2018 Revisión: 3/9/2018 Aceptación 17/1/2019 


\section{Introducción}

Durante mucho tiempo las personas con capacidades distintas tanto físicas como cognitivas han tenido trabas a la hora de buscar empleo, ya que se ha tenido la falsa creencia de que no tienen la aptitud o la habilidad para desarrollarlo de manera óptima. Sin embargo, esto no es verdad, ya que estas personas tienen la capacidad de desenvolverse en el trabajo de igual forma que una persona con capacidades dentro de la norma. A pesar de esto se les complica encontrar un trabajo estable debido a que sigue existiendo un recelo al contratar a personas con distintas capacidades. Conjuntamente es importante señalar que como todas las personas, las personas con capacidades distintas no pueden desarrollarse en cualquier trabajo, pero sí en trabajos de acuerdo a sus habilidades y destrezas.

En el Código de Trabajo de Costa Rica, publicado en agosto de 1943, en el apartado Igualdad de oportunidades para personas con discapacidad en el ámbito laboral, publicado por el Ministerio de Trabajo y Seguridad Social de Costa Rica, menciona:

Las personas trabajadoras con discapacidad, tienen los mismos derechos y deberes que cualquier otra persona trabajadora, por lo que se rigen de acuerdo al Código de Trabajo, Convenios Internacionales y otras leyes relacionadas con el mundo del trabajo (condiciones de trabajo justas y favorables, salarios mínimos, igual salario por trabajo de igual valor, condiciones de trabajo seguras y saludables, protección contra el acoso y la discriminación, derecho a sindicalizarse, a la capacitación, entre otros). (p. 1).

Las leyes que protegen los derechos de las personas con discapacidad, en donde están incluidas las personas con diversidad funcional cognitiva, según lo que dicta el Código de Trabajo de Costa Rica (1943) son:

- Ley 7600 de Igualdad de Oportunidades para Personas con Discapacidad.

- Ley 8661 Convención Internacional de Derechos de las Personas con Discapacidad

- Ley 8862 Inclusión y Protección laboral de las Personas con discapacidad

- Ley 7092 Ley de Incentivo a favor de los empleadores que contraten personas con Discapacidad.

Por lo tanto la presente investigación tiene como objetivo indagar y analizar las posibilidades que tienen las personas costarricenses con diversidad funcional cognitiva dentro del ámbito laboral. Esto se realizará con el fin de identificar cuáles han sido sus 
experiencias a la hora de buscar un empleo, cómo han sido sus entrevistas de trabajo, el trato que les brindan y cómo se han logrado desarrollar dentro de este ámbito.

\section{Antecedentes}

A continuación, se presentan algunos artículos encontrados que tratan el tema a indagar en la presente investigación. Se encuentran aportes venezolanos, chilenos, españoles, que incluyen la discapacidad intelectual como motivo de investigación. Sin embargo las investigaciones nacionales encontradas, se refieren más a la discapacidad física que a la cognitiva, por lo que se puede notar un vacío en el tema.

Se consultó una investigación realizada por Delmastro y De Montes realizado en Venezuela en el 2015. Esta tenía como objetivo explorar las distintas probabilidades que ofrecen las Empresas de Producción Social (EPS), como proyecto educativo para el logro de la inserción socio-laboral de personas con diversidad funcional cognitiva del Taller de Educación Laboral Bolivariano Costa Oriental del Lago (TELBCOL). Este consistió en un estudio de metodología cualitativa en donde se utilizó la modalidad investigación acción participante. Se utilizaron técnicas como la observación y la entrevista semi-estructurada

para la recolección de los datos. Los sujetos participantes fueron 169 jóvenes de ambos sexos con dificultades de aprendizaje, autismo, retardo mental o combinados, además se trabajó con el personal docente de la institución, padres de familia entre otros (Delmastro y Villegas, 2015).

Como resultados encontrados se concluyó que de las tres modalidades de empleo que se plantean en la investigación: la cooperativa, la microempresa y la empresa familiar, la última es la que logra ofrecerle mayores posibilidades a las personas con diversidad funcional cognitiva. Esto en virtud del acompañamiento y apoyo que puede brindar el grupo familiar en su consolidación y seguimiento, lo cual es de suma importancia para el desarrollo de las personas con diversidad cognitiva a la hora de conseguir empleo. Como sociedad este apoyo a estas personas queda debiéndose, debido a que se ve como un "problema" familiar que debe de resolverse en ese ámbito y no fuera de él (Delmastro y Villegas, 2015). 
Sin embargo, en Chile la situación de la inserción laboral de personas con alguna discapacidad intelectual es complicada, debido al tabú que sigue existiendo con respecto a la capacidad de estas personas. Como se muestra en un estudio bibliográfico de Vidal y Cornejo (2012) ellas mencionan que para esta población, aunque sean egresados de centros educacionales de formación laboral, la mayoría no logra insertarse adecuadamente en el mercado laboral y los empleadores no encuentran en estos el perfil requerido

Además, según la gravedad que presente la persona en su discapacidad intelectual le será más difícil adquirir un empleo, puesto que los que son mayormente empleados muestran una discapacidad leve. Vidal y Cornejo (2012) mencionan que se ha explicado y comprendido esta baja en la inserción de jóvenes con discapacidad intelectual en Chile, primero por la formación de las escuelas y centros de educación intelectual del país, que no se ajustan a las necesidades de las empresas. Segundo, por la falta de normativas que velen por la inserción laboral de esta población; tercero, porque se necesita un mayor financiamiento del país como apoyo a las empresas para la inserción laboral; cuarto, mayor compromiso social por parte de las empresas privadas. Quinto, por el déficit en programas para el apoyo de inserción laboral en la empresa de los jóvenes con discapacidad intelectual; y por último el que existe un alto nivel de desempleo general en el país: 7,0 \%.

Vidal y Cornejo (2012) mencionan diferentes programas que se han creado para el apoyo de personas con discapacidad intelectual, sin embargo muchas de estas han surgido en Europa. Ejemplo de esto lo es el Empleo con Apoyo (EcA) que es una metodología de inserción laboral para personas con discapacidad. De la misma forma también está la Asociación de Personas para el Empleo con Apoyo (APSE), sin embargo en Chile no se desarrolla con éxito como en Europa.

Los autores plantean que en Chile, la implementación del modelo de EcA necesita la transformación o generación de nuevas normativas en cuanto a la educación, salud, previsión social y trabajo, propiciando una preparación adecuada a los jóvenes con discapacidad lo más tempranamente. Debe incluir a la vez a la familia en orientaciones, apoyos y compromiso formativo, que permita el desarrollo de habilidades sociales y conductas adaptativas que potencien su autonomía y vayan maximizando el desarrollo de todas sus capacidades (Vidal y Cornejo, 2012). 
Millán (2002) realizó un estudio bibliográfico en Coruña, España, en donde recalca que el trabajo es importante en el desarrollo personal y mental de las personas con discapacidad intelectual. Esto debido a que causa en la persona satisfacción personal al poder desempeñarse en una labor donde se valoren sus esfuerzos. Al tener remuneración por su labor adquiere a la vez, más libertad de obtener objetos que desee, conjuntamente ayuda en su desarrollo psicomotriz y en las habilidades sociales.

Sin embargo, existen muchas limitantes para estas personas, tanto porque en los centros educativos no se les da el proceso necesario, así como porque en las empresas no se les brinda una inserción adecuada. Es decir existen muchas trabas que no permiten a estas personas desenvolverse en el área laboral (Millán, 2002).

Otro aspecto importante y que en ocasiones no se toma en consideración es la vejez, Millán (2002) menciona cómo para las personas con discapacidad intelectual a los 45 años se manifiestan los primeros signos de la vejez, por lo que para antes de esta edad estos comienzan a no ser consideradas productivas, siendo su desarrollo laboral menor en comparación a los demás. También, ante esto las empresas deben de ser cuidadosas al momento de despedir a estas personas, ya que su proceso es diferente, se debe de ir disminuyendo su trabajo paulatinamente, sin que la persona se sienta inútil.

La investigación de carácter cualitativa realizada por Rodríguez (2010) en Costa Rica, donde la investigadora estuvo inmersa en las experiencias de los y las participantes. Se utilizó como método de recolección de datos la revisión bibliográfica como primera parte, seguida de entrevistas a dos Licenciados en Derecho con experiencia en el campo laboral y cuatro entrevistas a profesionales en Educación Especial quienes se encontraban trabajando en tercer ciclo de educación especial, cuarto ciclo de educación especial y el programa de atención integral a personas adultas con discapacidad (CAIPAD).

Según esta investigación, como parte de las habilidades y destrezas que se les enseña a los/as niños/as y los/as adolescentes en los centros de educación especial del país, Rodríguez (2010) menciona que se encuentran:

1. Seguimiento de instrucciones.

2. Ajuste a un horario.

3. Uso de uniforme.

4. Aplicación de normas de seguridad. 
5. Sistema de comunicación de uso funcional (verbal o no verbal).

6. Tolerancia a tiempos de espera.

7. Tolerancia a cambios de rutina.

8. Ubicación de los materiales de trabajo (para buscarlos y para guardarlos).

9. Hábitos de aseo e higiene personal.

10. Normas de cortesía y buenos modales.

11. Independencia al momento de alimentarse y utilizar el servicio sanitario.

12. Reconocimiento de sus pertenencias.

13. Compartir con los demás.

14. Ajustarse a su espacio de trabajo y mantenerlo aseado.

15. Autorregulación y control de impulsos.

16. Toma de decisiones.

17. Uso del tiempo libre.

18. Desplazamiento independiente.

19. Reconocimiento de situaciones de peligro y acciones tendientes a su protección. Uso de servicios de transporte (si es necesario que los utilice).

20. Habilidades académico funcionales: ubicación temporo-espacial (días de la semana, actividades por días), lectura del reloj, conteo, lectura, escritura, reconocimiento de rótulos, uso del dinero. Esto según el oficio o tarea que se le asignará.

21. Desarrollo de tareas específicas según el empleo al que se pretende integrar y las funciones que en dicho puesto se le asignen.

Además se menciona que se deben dar ajustes y apoyos a las personas con discapacidad en el ámbito laboral de igual manera como se hace en el ámbito educativo. En lo que a ajustes y apoyos se refiere se definen dos tipos de adaptaciones que se ponen en práctica para lograr la participación de las personas con discapacidad en igualdad de condiciones, según sus características y necesidades. Estas adaptaciones son utilizadas en su mayoría de veces en Educación, los cuales son: las de acceso y las curriculares significativas y no significativas (Rodríguez, 2010).

Como se puede notar por las investigaciones presentadas, hay información importante sobre el tema en distintos países de Latinoamérica y Europa, que aportan a la 
presente investigación, dando una guía sobre lo que sucede con estas personas al entrar en el ámbito laboral. Existe cierto recelo por parte de los empleadores muchas veces de contratar personas con discapacidad cognitiva o con capacidades diferentes, esto causa que para esta población sea difícil poder insertarse dentro del ámbito laboral y realizarse como persona a nivel económica y personal. Es por este motivo que es de suma importancia la realización de esta investigación, para lograr dar a conocer las experiencias de inserción laboral de personas con diversidad funcional cognitiva del cantón de San Ramón. Además, se ve como una alternativa metodológica la cualitativa puesto que con esta se puede obtener el relato de las personas, aportando cuáles han sido sus experiencias y aprendizajes respecto al tema.

\section{Marco teórico}

En este apartado se explicarán a fondo los términos que son de importante relevancia dentro de la investigación. Para esto se realizó una revisión bibliográfica encontrando varios artículos que apoyan teóricamente este estudio. Los términos que se van a definir son: discapacidad, diversidad funcional, diversidad funcional cognitiva, discriminación, derechos y deberes laborales de las personas con diversidad cognitiva, inserción laboral, y exclusión laboral.

\subsection{Discapacidad}

La Organización Mundial de la Salud (2011), explica cómo la discapacidad es una condición que forma parte del ser humano, por lo tanto casi todas las personas en algún momento de la vida tendrán una discapacidad temporal o permanente y los que llegan a sobrevivir experimentarán gradualmente más dificultad en su funcionamiento. Además en la mayoría de las familias los integrantes sin alguna discapacidad ayudan a sus familiares que si la presentan, dando apoyo a estos.

Actualmente se ha buscado y luchado para que se considera la discapacidad dentro de los derechos humanos, puesto que tiempo atrás estas personas eran tratadas de manera segregada, buscando "soluciones" a los mismos. Sin embargo, se han cambiado políticas, buscando la inclusión reconociendo que la discapacidad en los seres humanos se origina tanto en los factores ambientales, como en el cuerpo (OMS, 2011). 


\subsection{Diversidad funcional}

$\mathrm{Al}$ hablar de la diversidad funcional cognitiva primeramente es importante entender la diversidad funcional, la cual según Palacios y Romañach (2006) es parte de un modelo en donde se basan en postulados de los movimientos de vida independiente y por lo tanto se ve a la persona con discapacidad o con diversidad funcional, como un ser valioso en sí mismo por su diversidad. Así la diversidad funcional cambia la percepción hacia las personas con alguna discapacidad, borrando la mirada de que estas personas son ineficientes y con un mal, sino que con sus diversidades se busca enriquecer, tomando la discapacidad como parte de los derechos humanos.

Conjuntamente se debe de señalar que aunque la diversidad funcional busca ver a todas las personas como seres valiosos por sí mismos, y tras las luchas llevadas a cabo por lograr este objetivo, existe una visión social de las mujeres y hombres con diversidad funcional diferente. Aún se dan caracterizaciones hacia estas personas haciendo que se creen disimilitud en relación con personas consideradas socialmente "normales".

Por otra parte, la Organización Mundial de la Salud menciona que el término Diversidad Funcional sugiere una visión mucho más genuina de la discapacidad hablando de diferentes capacidades y no refiriéndose a deficiencias, limitaciones ni restricciones. Así con la utilización de este término, se puede evitar que se creen diferenciaciones despreciativas como minusválido= menos válido (Ferreira, 2009).

El término persona con diversidad funcional, tiene su origen, según se menciona en el libro "Prisioneros del Cuerpo: La construcción social de la diversidad funcional" del autor Antonio Iañez (2009), en los debates que se establecen dentro del Foro de Vida Independiente-España. Este debate nace debido a que se realiza un análisis de los términos que se han utilizado durante todo este tiempo, para referirse a este sector de población. Algunos ejemplos de estos términos son: inválido, subnormal, deficiente, inútil, retrasado, idiota, minusválido, mutilado, lisiado, etc. A partir de este análisis, se llega a la conclusión de que todos estos términos muestran una afinidad ofensiva, de ahí que se da, en parte, la estigmatización que padece este grupo. 


\subsection{Diversidad Funcional Cognitiva}

La diversidad funcional cognitiva refiere a las personas con dificultades en el desempeño a nivel de una o varias de las funciones cognitivas, tanto en los procesos de entrada, elaboración y respuesta, que por lo tanto influyen en el procesamiento de información y en el aprendizaje, por lo que requieren apoyo para que mejoren su funcionalidad. Es relevante distinguir que la discapacidad cognitiva no refiere precisamente a diagnósticos como retraso mental o dificultades de aprendizaje, sino que refiere a la funcionalidad que alude al desempeño cognitivo de cualquier persona (Ministerio de Educación Nacional, 2006).

\subsection{Discriminación}

Con lo desarrollado anteriormente es necesario explicar qué se entiende por discriminación, que según la Comisión Nacional de los Derechos Humanos (2012) el acto de discriminar es seleccionar excluyendo, es decir tratar de manera inferior a las personas o grupos. Entre los motivos por los cuales se tiende a discriminar están, la etnia o nacionalidad, elecciones políticas y sexuales, la religión, el género, la edad, opiniones, estado civil, condiciones de salud, entre otras incluyendo la discapacidad.

Cuando se realiza la discriminación se hace en base a una distinción injustificada y arbitraria relacionada con las características de una persona o su pertenencia a algún grupo específico. En esta se ejecutan actos o conductas que impide a las personas la igualdad de trato, lo que ocasiona que se anule o restrinja el goce de sus derechos humanos. Cabe rescatar que aunque la discriminación ejecuta estos actos contrarios, todas las personas en esencia son iguales y gozan de los mismos derechos. Además se debe considerar que toda persona puede ser víctima de discriminación, pero son más discriminadas y afectadas aquellas que se encuentren en una situación de vulnerabilidad o desventaja, por una circunstancia social o personal (Comisión Nacional de los Derechos Humanos, 2012).

\subsection{Derechos y Deberes Laborales}

El Ministerio de Trabajo y Seguridad Social (s.f.) de Costa Rica enmarca como las personas con diversidad funcional cognitiva poseen los mismos derechos y deberes que cualquier otra persona trabajadora, rigiéndose de acuerdo al Código de Trabajo, Convenios Internacionales y otras leyes relacionadas con el mundo del trabajo. Así mismo tienen el 
derecho a la readaptación o reubicación, y cuentan con oficinas e instituciones donde pueden recibir asesoría y buscar empleo, estas son: Departamento de Intermediación de Empleo de la Dirección

Nacional de Empleo; La Unidad de Equiparación de Oportunidades para Personas con Discapacidad; Instituto Nacional de Aprendizaje (INA) y el Ministerio de Educación Pública (MEP).

Departamento de Intermediación de Empleo de la Dirección Nacional de Empleo.

Este se encarga de ofrecer servicios públicos de intermediación de empleo, los cuales se encuentran destinados a personas que están en condición de desempleo, subempleo o por otro lado, que están en búsqueda de mejores oportunidades laborales (Ministerio de Trabajo y Seguridad Social. 2017).

\section{Unidad de Equiparación de Oportunidades para Personas con}

\section{Discapacidad.}

Esta se encarga de asesorar a las personas con discapacidad o diversidad funcional, en cuestión de equiparación de oportunidades laborales para las mismas. Además maneja situaciones de discriminación por discapacidad y por la tutela del cumplimiento de los derechos laborales (Ministerio de Trabajo y Seguridad Social. 2017).

Por otro lado, esta unidad se encarga también de promover la responsabilidad social empresarial como una acción afirmativa en la contratación de personas con discapacidad. El motivo de esto es que se logre mejorar las condiciones de vida de estas personas y sus familias (Ministerio de Trabajo y Seguridad Social. 2017).

Instituto Nacional de Aprendizaje.

Algunas políticas que del INA que favorecen a esta población, estas se citan seguidamente. Estas fueron recolectadas de la página oficial de la institución.

- El Instituto Nacional de Aprendizaje promueve la igualdad de género entre mujeres y hombres, cerrando brechas en la formación profesional y en el marco de la mejora continua del Sistema de Gestión de Igualdad y Equidad de Género, desde un enfoque de derechos humanos; en cumplimiento de los requisitos legales y reglamentarios aplicables y suscritos por el INA, mediante la transversalización del enfoque de género en el Sistema Nacional de 
Capacitación y Formación Profesional, aportando al cambio cultural y al desarrollo nacional. Acuerdo No 007-2013-JD.

- Garantizar el respeto y la accesibilidad a toda la población; sean estas personas funcionarias, participantes o aquellas que accedan a la institución; promoviendo los ajustes que consideren los postulados de acceso universal y aseguren adaptaciones en lo actitudinal, información y comunicación, jurídico, administrativo, espacio físico, transporte, servicios y productos de apoyo; promoviendo la igualdad y equiparación de oportunidades. Acuerdo 250-2015 JD.

- El INA facilita con equidad e igualdad de oportunidades el acceso de la población en desventaja social a los servicios de capacitación y formación profesional, coadyuvando así en la movilidad social ascendente. Acuerdo 2392015-JD V2 Y GG-614-2015.

\subsection{Inserción Laboral}

Según la investigación de Rodríguez (2010) la inserción laboral se refiere al acompañamiento que se les brinda a las personas que lo requieran. Lo que se busca con la inserción laboral es facilitar su incorporación a un puesto de trabajo determinado, como parte del mercado laboral y la fuerza productiva de la sociedad.

Por su parte en la investigación realizada por Ordóñez (2011) se menciona que en el proceso de inserción laboral de una persona con alguna discapacidad se deben de tener en cuenta las medidas que aseguren el acceso y la permanencia de la persona en su lugar de trabajo, manteniendo el respeto hacia su individualidad y al tipo de discapacidad que presente. El mismo autor indica que para lograr esta verdadera inserción laboral es necesario comenzar mejorando la educación y la formación ocupacional; siendo de suma importancia que se desarrollen competencias y capacidades en esta población, para que se les facilite su inserción laboral.

\subsection{Exclusión Laboral}

El concepto de exclusión laboral se encuentra incluido dentro de un concepto más amplio de exclusión social entendido como un debilitamiento o quiebre de los vínculos que unen al individuo con la sociedad. De este modo, individuos que antes no se encontraban socialmente excluidos y que tenían una participación económica y socialmente activa pasan 
a quedar excluidos ante transformaciones socio-económicas en términos generales, o productivas en particular (Cedrés y Maillot, 2001).

Por otra parte, según el artículo publicado por la CEPAL en 2001, titulado "Procesos de exclusión e inclusión laboral: la expansión del empleo en el sector terciario", menciona que "muchas de las desigualdades e injusticias que aparecen en el proceso de desarrollo económico pueden ser interpretadas como exclusiones de los derechos básicos, de las instituciones sociales, de los mercados y de las oportunidades de ingresos, de representación y de participación” (Ruiz-Tagle, citado en Weller 2001).

Según el artículo realizado por Enriqueta Martín en 2005 y publicado en la página de la Asociación Síndrome de Down en 2010, menciona que entre los factores más visibles que impiden a las personas con diversidad intelectual un buen acceso al mundo laboral son:

- Un desconocimiento por gran parte de la sociedad del potencial de estas personas.

- Las pocas oportunidades que les ofrecemos para que demuestren de lo que son capaces.

- Y por último, y que no debemos olvidar, es que las personas con diversidad intelectual no pelean, ni se manifiestan, ni molestan al gobierno, ni a ninguna central sindical, ni al dueño de una empresa, ni siquiera a su familia, exigiendo sus derechos y la posibilidad de acceder a un puesto de trabajo como cualquier ciudadano.

\section{Objetivos}

\subsection{Objetivo General}

1. Reconocer la situación laboral de personas con diversidad funcional cognitiva en Naranjo de Alajuela, desde la perspectiva de un experto en el cuido y trabajo con personas con diversidad funcional cognitiva, de una persona con diversidad funcional cognitiva.

\subsection{Objetivos Específicos}

1. Identificar las problemáticas en la obtención de trabajo de las personas con diversidad funcional cognitiva, desde la perspectiva de de un experto en el cuido 
y trabajo con personas con diversidad funcional cognitiva y de una persona con diversidad funcional cognitiva.

2. Describir las facilidades en la búsqueda de trabajo de las personas con diversidad funcional cognitiva, desde la perspectiva de un experto en el cuido y trabajo con personas con diversidad funcional cognitiva y de una persona con diversidad funcional cognitiva.

3. Indagar sobre el proceso de inserción laboral de las personas con diversidad funcional cognitiva, desde la perspectiva de un experto en el cuido y trabajo con personas con diversidad funcional cognitiva y de una persona con diversidad funcional cognitiva.

\section{Metodología}

\subsection{Tipo de estudio}

Para la realización de esta investigación, se utilizó como metodología el estudio cualitativo, ya que esta recoge el discurso de las personas entrevistadas, y seguidamente se realizó una interpretación del mismo con base a lo que se busca con el estudio. Un punto importante de esta metodología es que las personas investigadoras estudian la realidad del caso, dentro de su contexto (Hernández, Fernández y Baptista, 2006). Conjuntamente la credibilidad de los métodos cualitativos se conforman de las habilidades, la competencia y el rigor de la o las personas que realizan el trabajo de campo; con esto los trabajadores de campo aprenden que ellos mismos son el instrumento principal y más confiable de observación, selección, coordinación e interpretación" (Arias et al, 2011).

Para la obtención de la información que sirva para cumplir con los objetivos, se hizo una revisión bibliográfica de los estudios realizados y teoría necesaria para la investigación. Seguidamente se aplicó una entrevista semiestructurada a dos expertos en el tema, el cual genera información actualizada, también se aplicó entrevistas a tres personas que presenten esta condición de diversidad funcional cognitiva.

\subsection{Participantes}

Para la ejecución de las entrevistas semiestructuradas se realizó el contacto con el CAIPAD de Naranjo, el cual busca brindar oportunidades del desarrollo de oportunidades u 
ocupaciones a personas con alguna diversidad tanto física como cognitiva. En esta institución se realizaron la entrevista a dos personas expertas en el trabajo y cuido de personas con diversidad funcional cognitiva, las cuales a su vez facilitaron el contacto con tres personas con diversidad funcional cognitiva que se encuentran en el centro en busca de trabajo y que estuvieron dispuestos a colaborar con la investigación.

\subsection{Criterios para garantizar la calidad de la información}

Los criterios de confiabilidad y validez que permite garantizar la calidad de la información, resultados y análisis es mediante la triangulación de la información. La información triangulada se obtendrá de los datos de las entrevistas de las personas participantes y la base teórica, ya que permite la creación de categorías y subcategorías que da la posibilidad de confirmar y comparar la información de las diversas fuentes.

Taylor y Bogdan (1984) afirman que para la realización de un análisis confiable es necesario crear categorías de análisis con la información que bibliográfica existente, organizándola y clasificándola. Así con las categorías ya establecidas se analiza la información producto del proceso. Este procedimiento corresponde a los criterios de credibilidad del paradigma interpretativo, que a su vez enriquece la validez interna, ya que permite deducir más allá de los datos y fenómenos, interpretando la complejidad de la realidad desde el contexto de la persona que lo vive.

\subsection{Criterios Éticos}

Los criterios éticos a tomados en cuenta dentro de esta investigación, fueron la confidencialidad que se les garantizará a los y las participantes con respecto a la información que nos brindaron, la cual estará dirigida únicamente a la realización del trabajo académico. Por su parte se les brindó un consentimiento informado en donde se les explicó lo que se va a realizar, cuánto será el tiempo de duración de las entrevistas, y se les aclaró que pueden retirarse en cualquier momento ya que no están obligados a participar.

\section{Resultados}

Se realizó el contacto con los CAIPAD, lo cual se trató de llamar a cuatro de ellos, teniendo solo respuesta del CAIPAD de Naranjo, que debido a la dificultad de contactarlos este proceso tomó aproximadamente tres meses. En este centro se realizaron dos entrevistas a expertos encargados de personas con diversidad funcional cognitiva y tres entrevistas a 
hombres con diversidad funcional cognitiva, del CAIPAD Talita Cumi Naranjo, entre los que estaban una persona con Retraso mental leve, uno con Trastorno orgánico de la conducta y uno con Esquizofrenia emocional. Cabe señalar que los entrevistados fueron seleccionados por la trabajadora social, considerándolos como adecuados para contestar la entrevista.

Persona A, presenta una condición de retardo mental leve, 40 años.

Medicado con ritalina, clonacepan y respiridona. Cuenta con primaria incompleta. "Sí fui pero no pude aprender lo que necesitaba aprender".

Ha laborado en el pasado en un supermercado por algún tiempo sin embargo tenía problemas con los compañeros y renunció a petición de su madre. "Yo lo que hacía era acomodar mercadería, después me ponía a ayudarle al gerente a atender a los clientes". "Me gustaba porque yo iba aprendiendo poco a poco". "Cuando estaba en bodega yo necesitaba que me dijeran que hacer, que me dieran indicaciones". Menciona que antes de trabajar en el supermercado, trabajaba en un llantero, "Mejor no les cuento lo que pasó (...) casi me violan, porque al muchacho le gustaba hacerme cosas, y yo lo reporté aquí y me sacaron de ahí".

Trabajó después en una veterinaria, de la cuál fue despedido después de 5 años por petición de los clientes debido a su condición, indica no le dieron liquidación. Menciona que él sabía qué tenía que hacer porque el dueño le mencionaba cada día sus tareas, sin embargo se le exigía mucho más allá de su capacidad, indica además en este lugar era maltratado por sus compañeros de trabajo, y sufrió de varios intentos de abuso sexual.

Se encuentra en el CAIPAD desde los 14 años. Y en este momento se encarga de la soda de la institución donde obtiene ganancias a partir de lo que compren sus compañeros, él lleva la contabilidad y saca sus ganancias, sin embargo las mismas se las entrega a su madre.

Persona B, Trastorno orgánico de la conducta, 25 años.

Cuenta con secundaria completa, así como bachillerato. Tiene 8 títulos de técnicos tanto del INA como de la misma organización (CAIPAD). Menciona haber intentado buscar trabajo pero que siempre le dicen que ellos lo llaman y nunca lo hacen. Varias veces comenta que su título no tiene valor debido a la leyenda que trae de haberse graduado de un colegio especial. Su sueño es trabajar como mecánico de autos, indica que le gusta mucho hacer 
dibujo. Es el encargado en su casa de realizar todos los mandados, considera que no tiene apoyo familiar necesario para buscar trabajo.

Menciona que tiene un problema y es el que no sabe leer ni escribir bien, sin embargo lo poco que sabe lo ha aprendido por sí solo. Trabaja en el centro de acopio del CAIPAD. "Buscar trabajo siendo una persona con discapacidad cuesta mucho". "Me han dicho que yo aparento ser una persona normal sin discapacidad, pero yo les digo que yo sí soy una persona normal". "Yo voy a seguir demostrando que yo sí puedo".

Persona C, Esquizofrenia emocional, 37 años.

Secundaria incompleta. Trabajó hace muchos años en una compañía recolectora de caña india, hasta que ésta quebró.

"Me gustaba mucho trabajar ahí, solo que quebró, después trabajé en otra empresa de caña y también quebró".

Estuvo en el programa Empléate por 10 meses, allí le daban una beca, sin embargo después de haber finalizado el proceso no le dieron empleo y pasó un año para que pudiera volver a ingresar al CAIPAD. "Nos dijeron que nos iban a conseguir un trabajo pero no nos dieron". "Mientras nos capacitaban nos daban una especia de ayuda".

Ve como una dificultad su edad, a la hora de conseguir trabajo, si busca lo dejan esperando.

"Por mi edad ya se me dificulta un poco conseguir trabajo".

"He ido a pedir trabajo, me han dicho que me llaman y no lo hacen".

Menciona que le encantaría trabajar si se le presentara la oportunidad. Cuenta con varios cursos que se imparten en el CAIPAD, en este momento trabaja dentro del centro atendiendo los teléfonos y haciendo limpieza, y cuenta con una remuneración. Comenta que siente el apoyo de sus padres en todo momento; se siente feliz en el CAIPAD y más de trabajar allí. Nos indica que su sueño es ser misceláneo, "me gustaría ser misceláneo, me gusta mucho eso".

\section{Entrevistas a expertos}

Directora del CAIPAD Naranjo

La directora del centro posee tres años en el puesto sin embargo anteriormente se encargaba del área de proyectos. Dentro de su experiencia menciona que su trabajo es muy enriquecedor, logrando conocer que los usuarios posee muchas cualidades, potencialidades 
y se llenan de frustración por no encontrar trabajo. Indica que la población se encuentra en su mayoría en edades de 35 a 55 años. Existen personas que poseen muchos años de estar ahí, y que se pueden emplear pero por sus discapacidades las personas no los emplean y además por ser Naranjo una zona cafetalera y con poca industria y a está le da miedo contratar, sintiéndose los usuarios discriminados. Mencionada además, que si hay como tres personas que han salido del CAIPAD y están laborando, pero con frecuencia la población se contrata como por periodos. En el centro se encuentran personas con discapacidades físicas, mentales, síndrome de down, parálisis cerebral infantil y degenerativa, predominando retardo mental, la cual casi nunca es contratada.

Menciona que existe todo un documento del código de trabajo, que "es muy bonito en papel", pero solo hasta ahí, y el ministerio de trabajo y los pre-vocacionales ayudar a la inserción laboral y también los CAIPADS "pero es muy complicado”. Igual la inserción se da por "el pobrecito", "al tontito hay que darle trabajo" y otros han sido burlados, agarrados como "bufón", siendo muy discriminatorio, incluso ha tenido experiencias de hablar con personas que trabajan en el área de discapacidad y han expresado que no contrataría a nadie con discapacidad, siendo este discurso muy contradictorio. Además, la directora comenta que la gente por la prisa no concuerda con la atención de estos, puesto su tiempo difiere al de los demás. También una de las dificultades es que a las personas se les paga muy mal, ya que "se piensa que ellos deben regalar su trabajo".

Ella piensa que las personas con discapacidad funcional cognitiva son muy capaces de laborar siempre con su medicación adecuada y en el trabajo adecuado, ya que no tienen funcionalidad en todo tipo de trabajo, también por su tipo de capacidades y de la historia de vida, y que para su inserción la persona tiene que ser consciente de que es todo un reto y debe tener paciencia y que "quiera aprender, no es que vaya enseñar, es que quiera aprender".

Entre los apoyos con los que cuentan las personas están el equipo de CAIPAD, de las residencias, de las empresas donde laboren (los que trabajan) y el apoyo de la casa, el cual es fundamental y a veces no se da. La motivación familiar es esencial en esta población, pues esta es lo que los impulsa o limita en su desarrollo. Además es de importancia considerar que ellos no solo tiene un diagnostico sino que tienen una historia que influye en su discapacidad, "una vida oculta". 


\section{Trabajadora social CAIPAD Naranjo}

Habla como al comenzar a trabajar con la población con discapacidad se le ofrece “el cielo y las estrellas", existiendo mucha reglamentación y leyes que los protegen pero al ejercerlas no funcionan, tanto que es el mismo sistema el que se encarga de sabotearlas y contradecirlas, que en el caso de ellos el mismo Ministerio de Educación funciona como obstáculo, comenta como a ellos se les brinda un folleto de que deben trabajar con la población, pero este se enfoca en personas menores de edad y ellos trabajan con adultos, contando hasta el momento con 60 matrículas, y que por lo tanto lo que les pide el MEP que trabaje con ellos son temas que las personas ya conocen y manejan. Ejemplos de los temas son: "comunicación: capacidad para transmitir y comprender información; cuidado personal: incluye habilidades relacionadas con el aseo, comida vestido e higiene personal; habilidades sociales: Habilidades relacionales con intercambios sociales con otros individuos para iniciar, mantener y finalizar una conversación”.

Con esto, ella menciona "lo que nosotros hacemos es jugar con esas cosas porque lo que el Ministerio de Educación te da para hacer no funciona, la ley de autonomía que ahorita está vigente y es un bum no la han concretizado y no la han aterrizado y no la han adaptado a la población”. Además menciona que otro obstáculo es la familia que también tiene una discapacidad, pues no saben cómo trabajar con las personas con discapacidad y poder darles la autonomía y la motivación, "los limitan”, "le cortan las alas"; pero que a su vez es culpa de la sociedad que se encarga de "los vuelve a devolver y los vuelve a meter en su saco", no hay conocimiento de cómo tratar a esta población.

En lo laboral hay un problema, haciendo mención que los beneficiarios del CAIPAD tienen el apoyo (subsidio) del CONADIS pero a la hora de que ellos entran a una empresa el apoyo se los quita, hasta la matrícula del CAIPAD, y si ellos salen del trabajo deben de hacer todo el proceso para que vuelva a tener su apoyo, por lo que el buscar trabajo se limita por el miedo de perder la ayuda que tienen. Además indica que "las empresas nos piden condiciones para las personas con discapacidad, no pueden ir en silla de ruedas porque no tienen las instalaciones para una silla de ruedas, no pueden ser no videntes porque no tiene las adecuaciones, solo puede ser cognitivo pero leve, no puede tener trastornos conductuales, no pueden ser trastornos orgánicos y no pueden tener 
esquizofrenia y entonces tampoco es mi población”. Y si hay una ley que los ampara pero el mismo sistema la sigue saboteando, trayendo todo abajo, y además piden condiciones para ingresarlo en el área laboral que la mayoría no cumple. Conjuntamente indica que lo que no está escrito ni se menciona es que "la sociedad es la que tiene la discapacidad y usted como sociedad es la que tiene que hacer las adaptaciones", por lo tanto todo lo que ellos forman en el centro, al salir los usuarios a la sociedad se los trae a bajo, "la ley es muy bonita pero no la cumplen y a usted no la exigen cumplirla" y que las empresas contratan solo para que las vean que poseen conciencia social, pero solo contratan a personas que tengan una prótesis y una discapacidad leve, pero que ojala cognitivamente este bien.

Con esto ella habla como existen personas que quedaron atrapadas en el sistema, que entraron a los 20 años y ya van a cumplir 65 y que después pasan a CONAPAM que los envían a un hogar de ancianos y no se dará el trato brindado ahí que los tienen en labores los cinco días a la semana. Estas personas son muy capaces de laborar, súper laborales, incluso en ocasiones valoran más el trabajo, solo que no se les da la incorporación necesaria, y se les limita sus capacidades. Así los mayores retos que enfrentan son la aceptación y aceptación, tanto familiar, laboral y socialmente.

En cuanto a la preguntas de que si conoce alguna empresa o institución que ayude en la inserción laboral, habla de que se había creado un convenio entre el Ministerio de Educación y el INA, pero esto desde su opinión no funciona ya que muchos de los usuarios no lo poseen y no saben ni leer ni escribir, y no se adecua los cursos a la población.

En la sociedad y en lo laboral esta población se considera "como pobrecitos, como especialitos", dándose comentarios en actividades que ellos participan como en desfiles, de “vienen los mongolitos, de pobrecitos" incluso en una ocasión una persona mencionó "vean donde va esos tontitos, que polvo más desperdiciado", en definitiva la sociedad los estigmatiza mucho. Una relación que han logrado es crear un vínculo con la escuela, que al inicio los niños se mostraban con miedo, pero ahora tiene una gran relación.

Alguna de la población cuenta con el apoyo familiar pero no toda, además cuentan con el apoyo del equipo del CAPAD y del CONADIS. Necesitan amor, reconocimiento, aceptación, autonomía y oportunidades principalmente. 


\section{Análisis}

Tras los resultados obtenidos en las entrevistas se evidencia que una de las mayores problemáticas a las que se enfrentan las personas con discapacidad funcional cognitiva es a la discriminación. Esto se evidencia en el sujeto B, el cual comenta que él no saca tiempo para buscar trabajo debido a que su título tiene una leyenda, que al ser de un prevocacional no es un título valido, y las personas no los contratan. Entonces existe una desvalorización de los esfuerzos y las capacidades de estas personas. Donde son considerados como lo menciona la trabajadora social, tanto por la sociedad como por los lugares de trabajo "como pobrecitos, como especialitos".

Entonces se encuentra que incluso son contratados por empresas solo para su propio interés para que se vea que poseen conciencia social, pero para esto solo se da la contratación de personas con "una prótesis y una discapacidad leve, pero que ojala cognitivamente este bien" (trabajadora social). Conjuntamente la directora señala como la inserción se da por la creencia que se les está ayudando y no porque aporten a la empresa, puesto que la inserción se da por "el pobrecito", "al tontito hay que darle trabajo". Igualmente como se ve que por este desprestigio a la población muchos "han sido burlados, agarrados como bufón" en los propios trabajos, ya que no se valora el desempeño que realiza, incluso los clientes son los que desprecian su trabajo que como lo señala la directora menciona que la gente por la prisa no concuerda con la atención de estos, puesto su tiempo difiere al de los demás; con lo que también una de las dificultades es que a las personas se les paga muy mal, ya que se piensa que ellos deben regalar su trabajo. Esto se evidencia en el caso del sujeto A, que es despedido de su trabajo ya que no cumplía con a tiempo las exigencias del jefe que quería todo lo más rápido posible y por las quejas de los clientes, al decir que este tenía un tono de voz muy fuerte y que era molesto. Además por la vulnerabilidad ellos tienen mayores peligros en el trabajo, que en este mismo caso el sujeto A sufrió acoso sexual, tanto que se debió sacar de donde laboraba porque existieron muchos intentos de violación, por la consideración de que son "mongolitos" y que la gente incluso expresa "vean donde va esos tontitos, que polvo más desperdiciado" (trabajadora social).

Es decir, se evidencia el rechazo que recibe la población con discapacidad, pero aún es más propensa a la discriminación y a la dificultad de encontrar trabajo las personas con discapacidad funcional cognitiva puesto que se tiene menos conocimiento y más temor 
haciéndolas mayormente vulnerable. Inclusive se ve que sufren de peligros laborales y sociales como el acoso que, ya que incluso dejan de considerarse como personas sentipensantes.

Esta situación en las empresas y la concepción social de esta población da paso a analizar otra de las problemáticas que hacen a esta población ser acreedoras de la dificultad de encontrar trabajo y ser más propensas a contingencias, esto es el cumplimiento de las leyes que dicen protegerlos y amparar los. La directora del centro habla de cómo existe un documento (el código de trabajo) que es "muy bonito en el papel”, pero que este no se hace cumplir por lo que todo queda en palabras escritas. Que si bien el ministerio de trabajo y los pre-vocacionales buscan ayudar a la inserción laboral, al igual que los CAIPADS pero en su mayoría existen muchas dificultades. Así mismo la trabajadora social menciona "la ley es muy bonita pero no la cumplen y a usted no le exigen cumplirla"; también añade que aunque estas leyes los amparan, el mismo sistema es el encargado de sabotearlo.

La inserción laboral es complicada, ya que piden una serie de condiciones para el ingreso, condiciones que esta población queda excluida, pero lo que no se habla ni menciona es que "la sociedad es la que tiene la discapacidad y usted como sociedad es la que tiene que hacer las adaptaciones", es decir, no se deberían de dar estas condiciones para la inserción laboral, sino que más bien debería existir una reestructuración social que se encargue de hacer las adaptación para estas personas y así dar paso a la aceptación.

Otro aspecto que funciona como un factor de dificultad en la inserción laboral de las personas con discapacidad funcional cognitiva es la familia, que al igual en ocasiones sirve de apoyo. La trabajadora social habla de cómo la familia en ocasiones es un obstáculo, ya que esta también tiene una discapacidad, al desconocimiento de cómo debe de trabajar o interactuar con las personas con discapacidad y poder darles la autonomía y la motivación, en muchos casos "los limitan", "le cortan las alas"; aunque con esto ella analiza y expresa que esta situación de igual forma es reflejo de la sociedad que "los vuelve a devolver y los vuelve a meter en su saco", no hay conocimiento de cómo tratar a esta población.

Aunque si buen esta es un obstáculo, como se mencionó anteriormente esta puede ser un apoyo que como lo menciona a directora, este es fundamental, la motivación familiar es esencial en esta población, pues esta es lo que los impulsa o limita en su desarrollo. Conjuntamente estos tiene otros recursos que son facilitadores en la búsqueda de trabajo y 
la inserción laboral que es el apoyos brindado por las personas que integran el equipo del CAIPAD, el de las residencias, y su permanencia en el área laboral tiene gran peso el soporte de las empresas donde laboren (con los que trabajan). También un punto importante que señala la directora y que en ocasiones no se es tomando en cuenta y valorado es que ellos no solo tiene un diagnóstico sino que tienen una historia que influyen en su discapacidad, "una vida oculta" y que eso la mayor parte del tiempo se deja de lado. Así la mayor falta que tienen estas personas son "amor, reconocimiento, aceptación, autonomía y oportunidades" (trabajadora social).

\section{Conclusiones}

Como se puede observar a través del trabajo, las leyes para la igualdad de oportunidades laborales para personas con diversidad funcional existen, sin embargo estas no se aplican como deberían, o no están adecuadas realmente a la situación de estas personas. Además como lo mencionaba una de las entrevistadas, las leyes no se perciben igual para todas las personas, sino que cada jefe la aplica según sea su conveniencia.

Las leyes para la inclusión de personas con discapacidad o diversidad se adecúan mejor para personas con diversidades físicas y no tanto para diversidades cognitivas.

Por otro lado, a pesar de que se les brinde una oportunidad laboral, estas personas se ven expuestas a discriminación por parte de compañeros de trabajo, clientes, e incluso en algunas ocasiones, del mismo jefe. Como se puede observar en la primera entrevista en donde el sujeto tenía problemas con sus compañeros de trabajo por discriminación, y fue despedido del mismo a petición de clientes que no aceptaban su diversidad.

Además de lo ya mencionado, se encontró que existen instituciones que son creadas o especializadas en ayudar en la parte laboral a esta población, por ejemplo el INA o Empléate. Sin embargo, estas desde la percepción de los y las entrevistadas no brindan por completo la ayuda necesaria para lo que se van a encontrar al buscar empleo, como el INA, el cual capacita a estas personas pero no les ayuda con la inserción laboral en distintos campos, como lo vemos en el caso del joven de 25 años quien tiene varios técnicos del INA pero se encuentra desempleado. 
Como otra conclusión está el miedo al rechazo que se les ha infundido socialmente a esta población, por lo cual muchos ni siquiera hacen el intento de buscar un trabajo porque no lo van a obtener debido a su condición, la cual es vista como "anormal".

Finalmente se concluye que según lo que se les pide como requisitos al CAIPAD para contratar personas con diversidad, es o se ve como necesario dentro de las empresas que estas personas se adapten al funcionamiento de las mismas, en vez de ser ellos quienes se adapten a las diversidades de las personas.

\section{Referencias}

Arias, M., y Giraldo, C.V. (2011) El rigor científico en la investigación cualitativa. Investigación y Educación en Enfermería, 29, (3). Medellín, Colombia.

Cedrés, M., y Maillot, M (2001) Exclusión- Inclusión Laboral. Zona Económica, Uruguay. Recuperado de: http://www.zonaeconomica.com/exclusion-inclusion-laboral.

Comisión Nacional de los Derechos Humanos. (2012). La discriminación y el derecho a la no discriminación. México. Recuperado de: http://www.cndh.org.mx/sites/all/doc/cartillas/2_Cartilla_Discriminacion.pdf

Delmastro, A.,y Villegas, M. (2015) Empresas de producción social como alternativa para la inserción Socio-Laboral de las personas con diversidad funcional cognitiva. Revista Arbitrada Venezolana del Núcleo Luz-Costa Oriental del Lago, 22-33.

Ferreira, M. (2010) De la minus-valía a la diversidad funcional: un nuevo marco teóricometodológico. Política y Sociedad. Universidad Complutense de Madrid. Vol. 47 Núm. 1: 45-65 45.

Hernández, R., Fernández, C. y Baptista, P. (2006) Metodología de la investigación, 4ta Edición. Mc Graw Hill. México.

Iañez Domínguez, A. (2009) Prisioneros del Cuerpo: La construcción social de la diversidad funcional. Diversitas Ediciones. Primera Edición. España.

Instituto Nacional de Aprendizaje (INA). Políticas Institucionales. Recuperado de: http://www.ina.ac.cr/institucional/

Martín, E. (2010) Exclusión laboral de mujeres y jóvenes con discapacidad. Asociación síndrome de Down (ASALSIDO) Artículo redactado en noviembre de 2005. 
Recuperado de: http://www.asalsido.org/asalsido/lista-art-profesionales/33-insercinlaboral/261-exclusion-laboral-de-mujeres-y-jovenes-con-discapacidad.html

Millán, J. (2002). Inclusión socio-laboral y envejecimiento en las personas con discapacidad intelectual. Importancia del trabajo para la integración social para las personas adultas con discapacidad. Coruña, España.

Ministerio de Educación Nacional. (2006). Orientaciones Pedagógicas para la atención educativa a estudiantes con discapacidad cognitiva. Colombia, Bogotá. Recuperado de http://www.colombiaaprende.edu.co/html/micrositios/1752/articles320691_archivo_5.pdf

Ministerio de Trabajo y Seguridad Social. (s.f.). Igualdad de oportunidades para personas con discapacidad en el ámbito laboral. Recuperado de: http://www.mtss.go.cr/temas-laborales/12_Discapacidad-ind.pdf

Ministerio de Trabajo y Seguridad Social (2017) Red Institucional de Transparencia. Recuperado de: http://www.mtss.go.cr/

Organización Mundial de la Salud. (2011). Informe Mundial sobre la Discapacidad. Recuperado de: http://www1.paho.org/arg/images/Gallery/Informe_spa.pdf

Ordóñez, C. (2011) Breve análisis de la inserción laboral de personas con discapacidad en el Ecuador ALTERIDAD. Revista de Educación vol. 6, num. 2. pp. 145-147. Universidad Politécnica Salesiana. Cuenca, Ecuador

Palacios, A., y Romañach, J. (2006). El modelo de la diversidad: la bioética y los derechos humanos como herramientas para alcanzar la plena dignidad en la diversidad funcional. Recuperado de: http://earchivo.uc3m.es/bitstream/handle/10016/9899/diversidad.pdf?sequence $=1$

Rodríguez, T. (2010). Las oportunidades laborales de las personas con discapacidad en Costa Rica según la Convención sobre los derechos de las personas con discapacidad (Tesis por el grado de Magister Scientiae). Universidad Estatal a Distancia. San José, Costa Rica.

Salazar, J., Guerrero, J., Machado, Y., \& Cañedo, Rubén. (2009). Clima y cultura organizacional: dos componentes esenciales en la productividad laboral. ACIMED, 20(4), 67-75. 


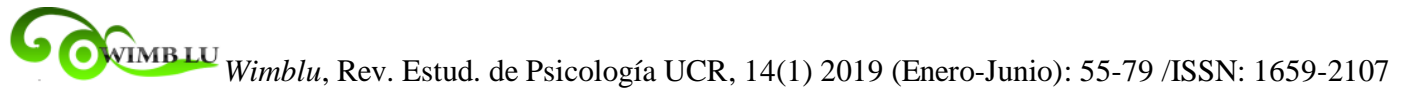

Taylor, S., y Bogdan, R. (1984). Introducción a los Métodos cualitativos de investigación. La búsqueda de significados. España: PAIDOS.

Tribunal Supremo de Elecciones (1943) Código de Trabajo. Publicado en la Gaceta No. 129. San José, Costa Rica.

Vidal, R., y Cornejo, C. (2012). Empleo con apoyo: una estrategia de inserción laboral para jóvenes con discapacidad intelectual $\mathrm{N}^{\circ} 1$. Facultad de Ciencias de la Educación. Universidad Católica del Maule. 113-127.

Weller, J. (2001) Procesos de exclusión e inclusión laboral: La expansión del empleo en el sector terciario. División de Desarrollo Económico. CEPAL. Chile.

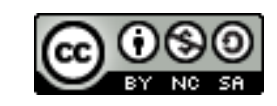

Esta obra está bajo una licencia de Creative Commons Reconocimiento-NoComercial-CompartirIgual 4.0 Internacional. 